\title{
How to Improve the Performance Prediction of a Pump as Turbine by Considering the Slip Phenomenon ${ }^{\dagger}$
}

\author{
Tommaso Capurso 1,*, Michele Stefanizzi 1, Marco Torresi 1, Giuseppe Pascazio ${ }^{1}$, \\ Giovanni Caramia ${ }^{1}$, Sergio M. Camporeale ${ }^{1}$, Bernardo Fortunato ${ }^{1}$ and Lorenzo Bergamini ${ }^{2}$ \\ 1 Department of Mechanics, Mathematics and Management (DMMM), Polytechnic University of Bari, \\ 70125 Bari, Italy; michele.stefanizzi@poliba.it (M.S.); marco.torresi@poliba.it (M.T.); \\ giuseppe.pascazio@poliba.it (G.P.); giovanni.caramia@poliba.it (G.C.); \\ sergio.camporeale@poliba.it (S.M.C.); bernardo.fortunato@poliba.it (B.F.) \\ 2 Nuovo Pignone, 70123 Bari, Italy; lorenzo.bergamini@bhge.com \\ * Correspondence: tommaso.capurso@poliba.it; Tel.: +39-327-915-0352 \\ + Presented at the 3rd EWaS International Conference on "Insights on the Water-Energy-Food Nexus", \\ Lefkada Island, Greece, 27-30 June 2018.
}

Published: 30 July 2018

\begin{abstract}
Nowadays Pumps working as Turbines (PaT) are devices widely used to perform energy recovery in hydraulic grids, thus improving their overall efficiency, and to build small hydropower plants. In this work, a centrifugal pump has been numerically investigated in turbine operating mode by means of the open-source CFD code OpenFOAM with emphasis on the flow field at the runner outlet. Due to the reduced number of blades in a PaT, the mean outlet relative velocity angle differs from the blade angle. In order to account for this phenomenon, the slip factor is introduced. The slip factor is investigated and its application to a 1D model is shown in order to highlight the improvement in predicting the characteristic curve of a centrifugal pump used in reverse mode as a turbine (PaT) especially at its part-load.
\end{abstract}

Keywords: PaT; slip factor; 1D model; OpenFOAM; CFD

\section{Introduction}

The world electricity demand is increasing year by year and the research of renewable energy sources instead of the fossil fuels attracts many investments. Large hydropower plants feed the national grid, whereas typical off-grid micro hydropower plants (MHP), in the range of 5-100 kW, are the most popular solutions for electrification among rural communities [1]. In MHP, hydraulic turbines are the critical technological component for energy production. Their cost has a great impact on the project budget, thus many researchers strongly suggest the use of PaT because they are mass-produced, cheaper than turbines and covers wide range of specific speeds and sizes. Nowadays, the prediction of the performance of a PaT is crucial in order to select the suited machine starting from its geometric information and its specific speed. The oscillations of the power produced by renewable sources is going to promote the need for energy storage such as micro hydro where the same machine needs to be operated both in pump mode and in turbine mode. Several models have been proposed for the prediction of the characteristic curve of a PaT; those based on statistic and normalization approaches are adopted when the geometric properties of the runner are unknown $[2,3]$ and are useful to evaluate the flow rate and the head exploited by the PaTs running at their BEP, whereas, the models based on the evaluation of the flow characteristics through the machine are used by constructors [4]. The latter models currently show a lack of accuracy in the prediction of the head vs flow rate curve 
especially at part loads and over loads. This work deals with the prediction of the performance of a $\mathrm{PaT}$, tested in both direct and inverse mode, by means of numerical simulations with emphasis on the flow field through the impeller. The computational domain reproduces the entire geometry of a commercial pump, including the suction pipe, the impeller and the discharge pipe. As shown by Ventrone [5] the relative velocity vectors at the outlet of a centripetal turbine, e.g., Francis turbine and $\mathrm{PaT}$, are subject to a deflection with respect to the blade congruent angle (slip). Ventrone [5] and Shi et al. [6] justify the slip phenomenon by demonstrating the presence of fluid flow vorticity between the blades in planes orthogonal to the rotational axis. Thus, counter rotating vortices appear inside the impeller channels. The proposed numerical investigation helps to describe this phenomenon. The introduction of a slip factor, oturbine, whose definition is equivalent to the one used for centrifugal pumps [7], accounts for the difference between the actual work and the theoretical one, due to the reduced number of the blades in PaTs compared to conventional turbines (e.g., Francis turbines) [5]. This analysis is based on the evaluation of the axial and tangential velocities at the mean radius of the trailing edge of the blades at the outlet of the centripetal impeller. Moreover, this analysis shows the presence of different phenomena, e.g., pressure gradient and secondary flows, which can occur at the outlet of a centripetal turbine and affect the performance of the machine. The definition of oturbine provides a great support in developing and tuning 1D models for the prediction of the turbine performance. Moreover, this analysis could provide the guidelines to successfully improve PaT geometries. A relationship between the new parameter (oturbine) and the mass flow rate is presented and discussed. Eventually, 1D models with and without the new corrections are applied and the deviations discussed [2,7-9].

\section{Numerical Methods}

The numerical investigations have been run with the open-source CFD code OpenFOAM by solving the 3D U-RANS equations. The unsteady RANS equations have been considered adequate in order to model the flow through the pump where quantities have to be considered averaged over a time period short enough with respect to global unsteady phenomena but long enough for statistical significance. The transient simulations have been run with an OpenFOAM application, pimpleDyMFoam, which is able to run transient simulations, including moving meshes, with incompressible flow. This application is based on the PIMPLE algorithm: a combination of the PISO (Pressure Implicit with Splitting of Operator) and SIMPLE (Semi-Implicit Method for Pressure-Linked Equations). The turbulence model applied for the system closure is the $k-\omega$ SST by Menter et al. [10]. This turbulence model is a standard to perform numerical analyses in hydraulic turbo machineries. It automatically uses the $k-\omega$ model in the near wall region whereas the $k-\varepsilon$ model away from the walls. The $k-\omega$ SST model can give an accurate prediction of flow separation explaining its common use for the numerical investigations of flow inside the centrifugal pumps [11].

\section{Numerical Domain and Boundary Conditions}

The geometry of a commercial pump with a specific speed $n_{q} \cong 21$ has been tested in both direct (pump) and inverse mode (turbine). The specific speed is defined as:

$$
n_{q}=\frac{n \sqrt{Q_{B E P} / f_{q}}}{H^{0.75}}
$$

where $H$ is the hydraulic head $(\mathrm{m}), Q$ is the flow rate $\left(\mathrm{m}^{3} / \mathrm{s}\right), n$ the rotational speed $(\mathrm{rpm})$ and $f_{q}$ is the number of impeller entries. The geometry studied in this work is represented in Figure 1. It includes the suction pipe, the impeller and the discharge. The selected configuration has a double suction impeller, actually two single suction centrifugal pump impellers in a back-to-back configuration. The geometry under investigation is composed by one double suction impeller with seven blades. This kind of centrifugal pump is employed to minimize the net positive suction head required when operating in pump mode. Moreover, the volute is double to balance the radial loads on the rotor and allow high-speed operation, also at part load [7]. 

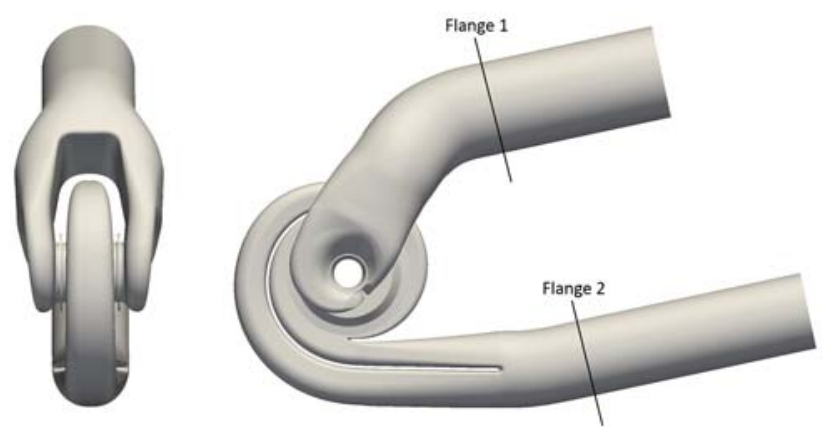

Figure 1. Front and side view of the CAD representing the centrifugal pump, areas of the Flange 1 and 2 equal to 0.02840 and $0.01941 \mathrm{~m}^{2}$, respectively.

For all the analyzed cases, the mass flow rate has been imposed at the inlet considering a uniform inlet velocity distribution. Moreover, the value of the mass flow leakage, which flows through the annular seal, has been modeled as exiting from the impeller case and incoming axially upstream the impeller eye with a $45^{\circ}$ of swirl with respect to the tangential direction. The leakage has been calculated a priori according to a one-dimensional empirical model because the geometry of the seal has not been included in the computational domain [7]. A uniform pressure distribution has been imposed at the outlet of the domain. At the inlet and outlet of the pump, straight pipes have been added to reduce uncertainties due to boundary conditions. The three parts of the geometry, which have been merged together, communicate each other by means of interfaces. In OpenFOAM the boundary condition used on these interfaces is named cyclicAMI (Arbitrary Mesh Interface). Furthermore, for this test case the turbulent intensity has been assumed constant and equal to $3 \%$ at the inlet of the domain. A critical aspect has been the definition of the wall roughness in OpenFOAM; the value of the equivalent sand grain roughness is equal to $5.6 \times 10^{-5} \mathrm{~m}$ and $5.6 \times 10^{-6} \mathrm{~m}$ respectively for the walls of the stator and rotor parts; the same values are used for all the geometries tested [12]. To do this the nutURoughWallFunction has been applied to the walls in the nut file together with $k q R W a l l F u n c t i o n$ and omegaWallFunction, respectively for $k$ and $\omega$ wall boundary conditions. Three different mesh sizes have been generated with the grid generator ICEM CFD ${ }^{\circledR}$ in order to perform a grid refinement study, see Table 1. Eventually, a grid made of 11 million of cells has been chosen because of small deviations in the results and a good compromise between the geometry refinement and the computational costs (see Table 1 and Figure 2).

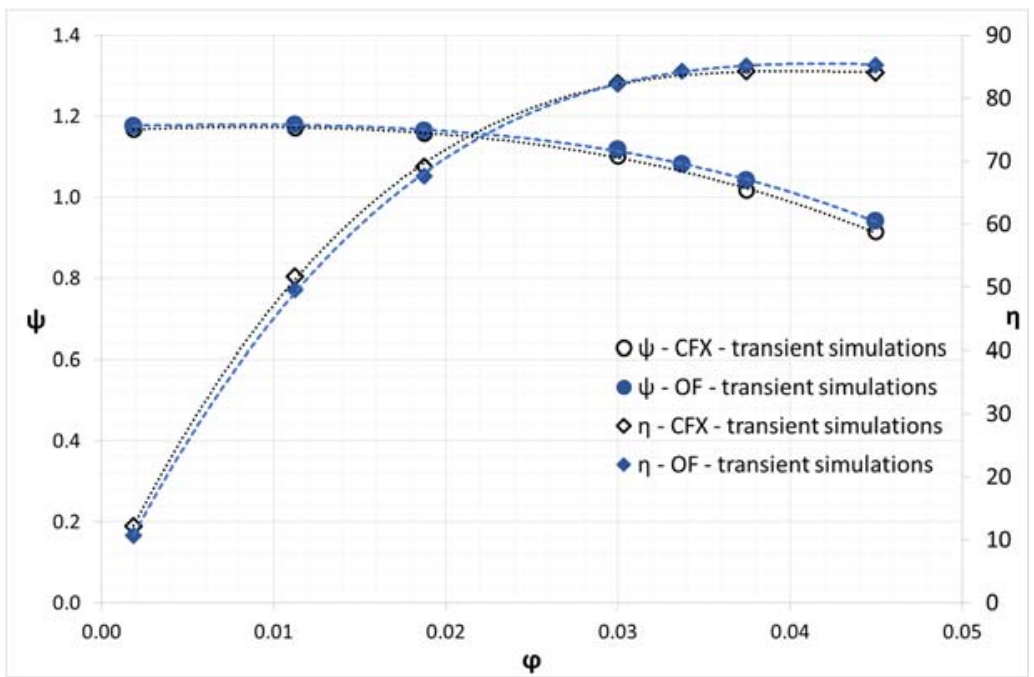

Figure 2. Comparison of the $\psi-\phi$ and $\eta-\phi$ curves calculated with CFX and OpenFOAM. 
Table 1. Different grid refinements with the pump Head calculated a t the BEP.

\begin{tabular}{cccc}
\hline & Coarse & Medium & Fine \\
\hline Impeller $\left(10^{6}\right)$ & 3 & 6 & 10 \\
Suction $\left(10^{6}\right)$ & 1 & 2 & 4 \\
Discharge $\left(10^{6}\right)$ & 2 & 3 & 6 \\
Total $\left(10^{6}\right)$ & 6 & 11 & 20 \\
Head at BEP $(\mathrm{m})$ & 139.8 & 140.8 & 141.0 \\
Time $(\mathrm{h}) 128$ cores & 7 & 18 & 40 \\
\hline
\end{tabular}

\section{Evaluation of the Machine Performance}

\subsection{Pump Mode}

Firstly, a numerical simulation of the flow through the machine (operating in pump mode) and a comparison of the results against the ones obtained by means of the consolidated commercial CFD code CFX has been performed. All the transient flow simulations have been carried out with a time step $\Delta t=T /(Z \times 256)=4.5 \times 10^{-6} \mathrm{~s}$ where $T$ is the time period of a complete rotation and $Z$ the number of blades for a duration total time equal to $0.085 \mathrm{~s}$, which corresponds to five complete impeller revolutions. The results in terms of head and efficiency have been averaged over the last 3 revolutions. The mean and the maximum values of the Courant number have been approximately equal to 0.03 and 6, respectively, in all the simulations. The results of the simulations for the pump mode are illustrated in Figure 2. These curves show a fairly good agreement with each other as already experienced by Nilson [13]. The results are illustrated by means of two dimensionless coefficients, the head coefficient, $\Psi$ :

$$
\Psi=\frac{2 g H}{u_{2}^{2}}
$$

and the flow coefficient, $\phi$ :

$$
\varphi=\frac{Q}{\pi D_{2}^{2} u_{2} / 4}
$$

In Equation (2), $u_{2}(\mathrm{~m} / \mathrm{s})$ is the tangential velocity calculated at the outer diameter $D_{2}(\mathrm{~m})$, whereas in Equation (3) $Q$ is the flow rate $\left(\mathrm{m}^{3} / \mathrm{s}\right)$.

The head, $H$, is evaluated as follows:

$$
H=\frac{P_{s t_{2}}-P_{s t_{1}}}{\rho g}
$$

where the subscripts 1 and 2 correspond to the inlet and outlet flanges, see Figure 1, whereas the efficiency, $\eta$, is defined as:

$$
\eta=\frac{\Delta P_{s t} Q}{C \omega}
$$

where the stagnation pressure variation, $\Delta P_{s t}$, is expressed in $\mathrm{N} / \mathrm{m}^{2}, C$ is the torque (Nm) resulting from the forces acting on the blades, hub and shroud of the impeller, comprising the disk friction losses, and $\omega$ is the rotational speed $(\mathrm{rad} / \mathrm{s})$.

\subsection{Turbine Mode}

Eventually a campaign of simulations has been carried out with the machine operated in reverse mode applying the same set up, previously described for the pump mode. In Figure 3 the characteristic curves of the impeller working either in pump or in turbine mode are represented on a $H / H_{B E P}$ vs. $Q / Q_{B E P}$ plane, where $H_{B E P}$ and $Q_{B E P}$ are the head and the flow rate at the BEP (Best Efficiency Point) of the pump. $H_{B E P, P} / H_{B E P, T}$ and $Q_{B E P, P} / Q_{B E P, T}$ are equal to 0.958 and 0.832 , respectively. PaTs work with higher mass flow rate than the same machine operating in pump mode. Therefore, numerical simulations have been performed starting from $Q / Q_{B E P, P}$ equal to $70 \%$, which is a value 
close to the runaway curve calculated according to the empirical model proposed by Gülich [7] (see Figure 3a), to $Q / Q_{B E P, P}$ equal to $140 \%$. The maximum flow rate allows us to identify the BEP of the turbine (see Figure $3 \mathrm{~b}$ ). The mass flow leakages have been calculated by means of the equations provided by Gülich [7] for centrifugal pumps. Applying this model the volumetric efficiency $\left(\eta_{v}\right)$ for a PaT results constant at various flow rate.

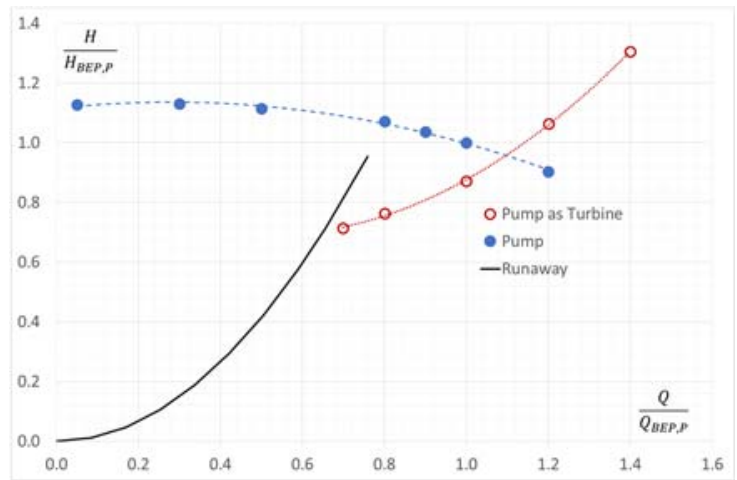

(a)

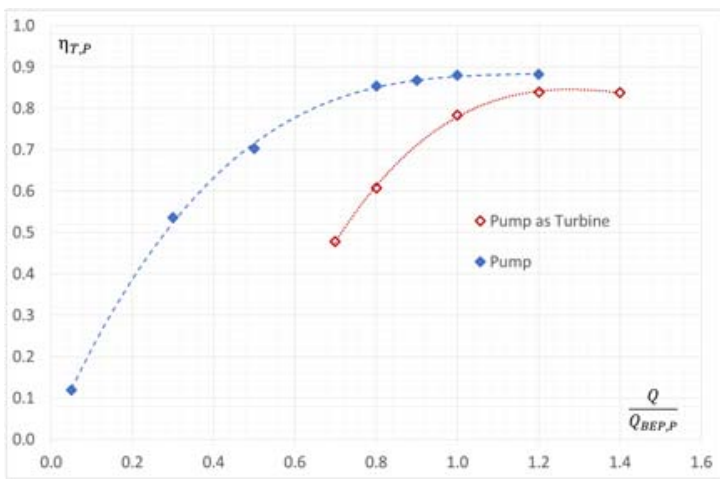

(b)

Figure 3. Plot of the hydraulic turbo machine performance in both pump and turbine operating modes: (a) Head curves calculated with Equation (4) with runaway curve; (b) Efficiency calculated with Equation (5).

\section{Results and Discussion}

As shown by Ventrone [5] and Shi et al. [6], under proper hypotheses, the fluid flow inside a blade channel in a rotating machine is subject to a counter rotating vortex with an intensity equal to:

$$
\bar{\nabla} \times \bar{w}=-2 \bar{\omega}
$$

Thus, vorticity is twice the angular velocity $(\bar{\omega})$ of the rotating machine (Equation $(6))$. This leads to a deviation of the relative velocity $\left(w_{1}\right)$ with respect to the blade congruent angle $\left(\beta_{1 B}\right)$ at the outlet of the impeller, see Figure 4.

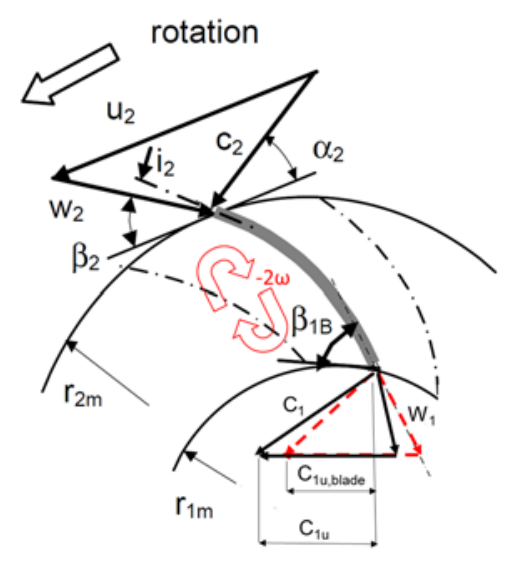

Figure 4. Velocity triangles at the inlet (2) and at the outlet of the runner (1). At the outlet both actual (solid line) and blade congruent (dashed line) velocity triangles are represented.

In Figure 4 both the actual and the blade congruent velocity triangles are represented. Actually, the main flow is subject to a deflection in the same direction of the machine rotation, which could be justified by the vorticity inside the channel. This deflection leads to an increase of the absolute tangential velocity component $(c u 1)$ at the outlet of the machine and therefore to a reduction of the work extracted by the runner according to Euler's equation (see Equation (7)).

$$
W=c_{u 2} u_{2}-c_{u 1} u_{1}
$$


With the aim to quantify this phenomenon, at the end of the numerical simulations, absolute tangential and axial velocity components have been evaluated at Section 1 , see Figure 4 , by means of area-weighted averages on surfaces downstream the impeller exit. These values have been averaged in time over the last 3 rotations of the machine. The mean value of the absolute tangential velocity is $4.40 \mathrm{~m} / \mathrm{s}$. This value has been compared with the theoretical value [14] computed at the same flow rate, congruently with the blade angle at the outlet of the impeller $\left(\beta_{1 B}\right)$. For the machine studied in this work, the theoretical tangential velocity, calculated by means of the blade congruent flow theory at the BEP of the turbine, is equal to $2.08 \mathrm{~m} / \mathrm{s}$. Furthermore, the presence of the blades induces a strong and localized deviation of the flow field. It has been noted that this might be justified by the pressure gradient that occurs between pressure and suction side of the blades (Figure 5). To point out this contribution, axial and tangential velocity components have been evaluated along the mean radius of the blades close to their trailing edge. The curve trends show a periodicity due to the presence of the blades (see Figure 6a,b).

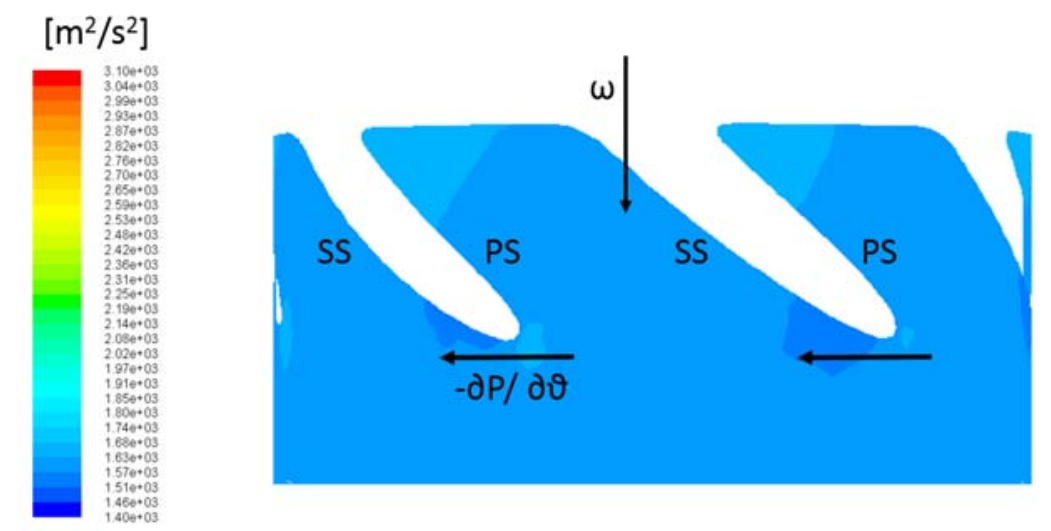

Figure 5. Contours of the static pressure $\left(\mathrm{m}^{2} / \mathrm{s}^{2}\right)$ evidencing the pressure gradient across the blade trailing edge at the mean radius.

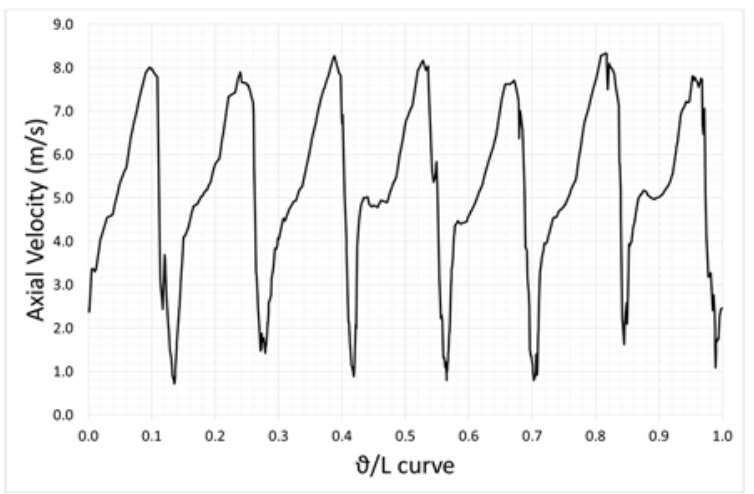

(a)

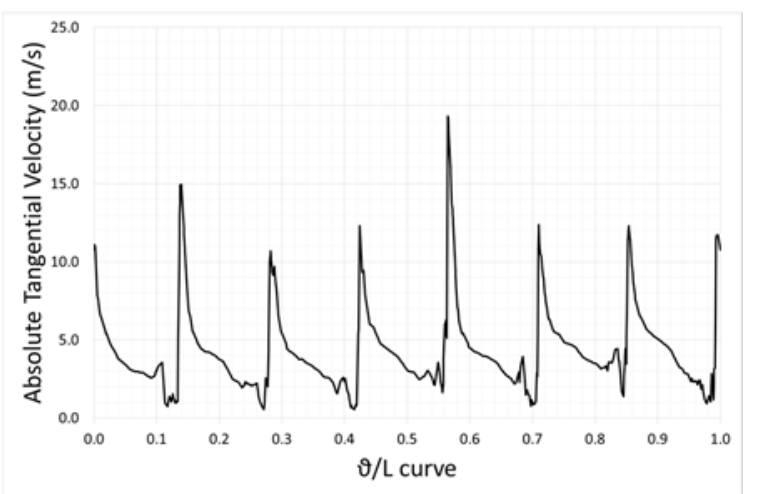

(b)

Figure 6. In this Figure, the velocity components of the absolute velocity at the outlet of the runner are represented: (a) Axial velocity along the mean radius at the trailing edge of the blade at the BEP; (b) Tangential velocity along the mean radius at the trailing edge of the blade at the BEP.

This means that the main flow at the outlet of the turbine is subject to a deviation not only due to the vortex inside the channel but also to the pressure gradient acting in the same direction. In order to describe the flow deflection at the outlet of a centripetal hydraulic turbo machine, the slip factor, oturbine, is introduced:

$$
\sigma_{\text {turbine }}=\frac{W_{C F D}}{W_{1 D \text { model }}}
$$

As previously described, the slip factor gathers different sources of deflection, e.g. counter rotating vortex and pressure gradient at the trailing edge of the blade. Applying the Euler's equation 
in order to compute the work both via $1 \mathrm{D}$ theoretical model, $W_{1 D}$ model, and CFD calculi, $W_{C F D}$, at the $B E P_{T}$, the $\sigma$ turbine is equal to 0.967 . Similarly to the theory of pumps where the slip factor corrects the ideal work transferred by the machine to the flow, the slip factor here introduced might be applied under turbine operating mode in order to correct the ideal work extracted by the PaT. The slip factor has been computed at different operating points, i.e., different mass flow rates, and the results are shown in Figure 7. Then, a model of the slip factor has been obtain by least-squares fitting.



Figure 7. Slip factor vs mass flow rates.

Numerical simulations at different flow rates are suggested for the purpose of further exploring the slip factor behavior. Eventually, three different slip factor models have been applied to the 1D model proposed by Stefanizzi et al. [15] to predict the PaT performance, see Figure 8 . The results show that by neglecting the slip factor $(\sigma$ turbine $=1)[2,7]$ the turbine head at the BEP is overestimated with an error of $+6.4 \%$. On the other hand, by applying either the Busemann's [8] or the Stodola's [9] model, the results at the BEP are in good agreement but the turbine head at part-loads is underestimated, with an error of $-8 \%$. Instead, by applying the least-squares fitting of the CFD results, as described in Figure 7, errors are minimized over a wide range of flow rates.

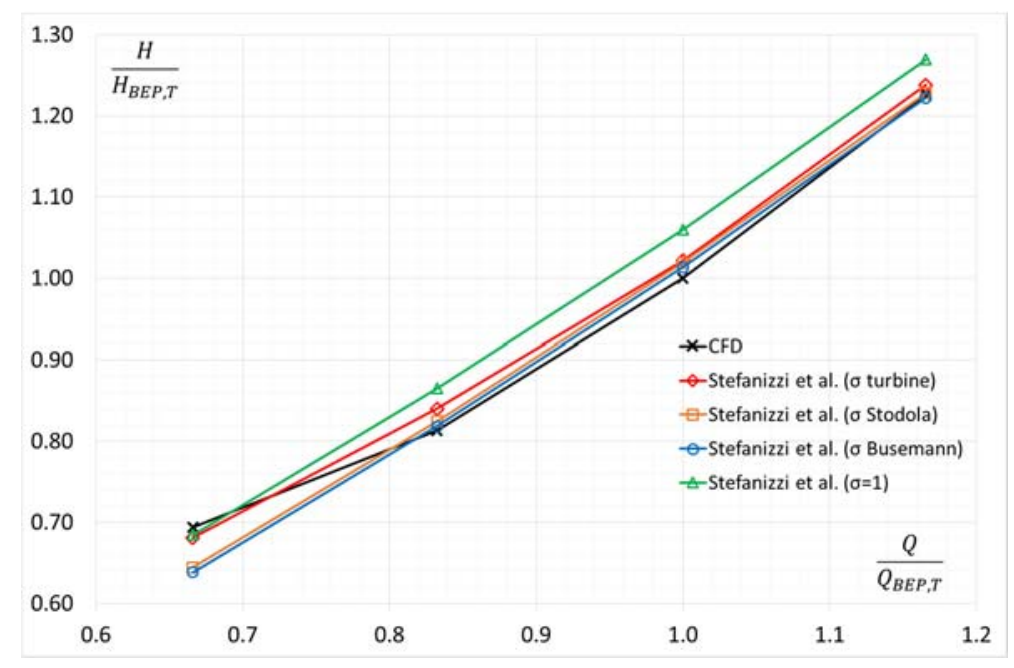

Figure 8. Comparison of different slip factor definitions (i.e., $\sigma$ turbine, $\sigma$ proposed by Busemann [8], $\sigma$ proposed by Stodola [9] and $\sigma=1$ ) with the CFD results.

\section{Conclusions}

This work shows numerical investigations of a centrifugal pump operating in reverse mode, namely working as a turbine, with emphasis on the flow field at the runner outlet with the aim to 
improve the accuracy of prediction model of PaT characteristic curves. The numerical analyses have been run by solving 3D U-RANS equations by means of the open-source CFD code OpenFOAM. According to Ventrone [5] and Shi et al. [6], the relative velocity vector at the outlet of the impeller shows a deflection in the direction of the peripheral velocity. This is due to the counter rotating vortex that is generated inside the impeller channels by Coriolis effect. In addition, another source of localized deflection is due to the pressure gradient that occurs at the trailing edge of the blades. The presence of these phenomena is pointed out by the evaluation of the tangential and axial absolute vectors components via the numerical simulation. These results have been compared with the data resulting from the blade congruent flow theory. This analysis allowed the introduction of the slip factor, $\sigma$ turbine, which has been helpful in order to improve a 1D model developed to predict the characteristic curve of a PaT starting from the pump geometry and its characteristic curves. Indeed, the proposed 1D model shows good results in a wide range of mass flow rates.

Author Contributions: T.C. wrote the paper, performed the CFD simulations and the data post-processing; M.S. developed the 1D model and conceived with T.C. the introduction of a slip factor in PaT; M.T. and G.P. supported the theoretical and numerical activities; G.C. helped with numerical set up and numerical simulations; S.M.C., B.F. and L.B. supervised the work.

\section{References}

1. Binama, M.; Su, W.-T.; Li, X.-B.; Li, F.-C.; Wei, X.-Z.; An, S. Investigation on pump as turbine (PAT) technical aspects for micro hydropower schemes: A state-of-the-art review. Renew. Sustain. Energy Rev. 2017, 79, 148-179, ISSN 1364-0321.

2. Yang, S.S.; Derakhshan, S.; Kong, F.Y. Theoretical, numerical and experimental prediction of pump as turbine performance. Renew. Energy 2012, 48, 507-513.

3. Derakhshan, S.; Nourbakhsh, A. Experimental study of characteristic curves of centrifugal pumps working as turbines in different specific speeds. Exp. Therm. Fluid Sci. 2008, 32, 800-807.

4. Barbarelli, S.; Amelio, M.; Florio, G. Predictive model estimating the performances of centrifugal pumps used as turbines. Energy 2016, 107, 103-121.

5. Ventrone, G. Deviazione Della Corrente Relativa Nelle Giranti Delle Turbine Francis; L'Energia Elettrica; 1972.

6. Shi, G.; Liu, X.; Yang, J.; Miao, S.; Li, J. Theoretical research of hydraulic turbine performance based on slip factor within centripetal impeller. Adv. Mech. Eng. 2015, 7, doi:10.1177/1687814015593864.

7. Gülich. J.F. Centrifugal Pumps, 2nd ed.; Springer: Berlin, Germany, 2008.

8. Busemann, A. Das Forderhohenverhaltnis Radialer Kreiselpumpen mit Logarithmisch-Spiraligen Schaufeln. Z. Angew. Math. Mech. 1928, 8, 372-384.

9. Stodola, A. Dampf und Gas-Turbinen; Springer: Berlin, Germany, 1924.

10. Menter, F.R.; Kuntz, M.; Langtry, R. Ten Years of Industrial Experience with the SST Turbulence Model. Turbul. Heat Mass Transf. 2003, 4, 625-632.

11. Huang, P.; Bardina, J.; Coakley, T. Turbulence Modeling Validation, Testing, and Development; NASA Technical Memorandum 110446; National Aeronautics and Space Administratio (NASA): Washington, DC, USA, 1997.

12. Adams, T.; Grant, C.; Watson, H. A Simple Algorithm to Relate Measured Surface Roughness to Equivalent Sand-grain Roughness. Int. J. Mech. Eng. Mechatron. 2012, 1, 66-71.

13. Nilsson, H. Nilsson Evaluation of OpenFOAM for CFD of turbulent flow in water turbines. In Proceedings of the 23rd IAHR Symposium, Yokohama, Japan, 17-21 October 2006.

14. Stefanizzi, M.; Capurso, T.; Torresi, M.; Pascazio, G.; Ranaldo, S.; Camporeale, S.M.; Fortunato, B.; Monteriso, R. Development of a 1-D prediction model of a PaT performance under design and off-design conditions. In Proceedings of the 3rd EWaS International Conference; Lefkada, Greece, 27-30 June 2018.

15. Stefanizzi, M.; Torresi, M.; Fortunato, B.; Camporeale, S.C. Experimental investigation and performance prediction modeling of a single stage centrifugal pump operating as turbine. Energy Procedia 2017, 126, $589-596$. 